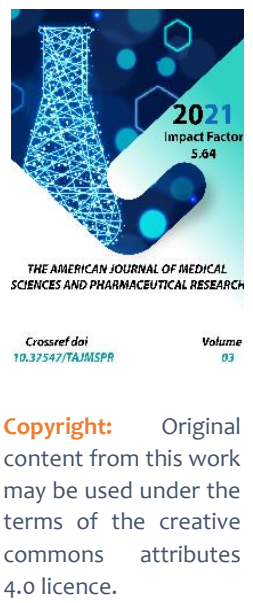

\section{Current Views On The Pathogenesis Of The Parietal-Visceral Pathway Of Gastric Cancer Metastasis}

Mirdjalol Dehkanovich Djuraev

Doctor Of Medical Sciences, Professor, Head Of The Department Of Oncology, Samarkand State Medical Institute, Uzbekistan

Nodir Maxammatkulovich Rahimov

Doctor Of Medical Sciences, Associate Professor, Department Of Oncology, Samarkand State Medical Institute, Uzbekistan

Mavluda Nigmatovna Karimova

Doctor Of Philosophy In Medical Sciences (PhD), Associate Professor At The Department of Oncology, Samarkand State Medical Institute, Uzbekistan

Shakhnoza Shavkatovna Shakhanova

Assistant In The Department Of Oncology, Samarkand State Medical Institute

Samarkand Branch Of The Republican Specialized Scientific And Practical Medical Centre, Uzbekistan

\title{
ABSTRACT
}

Metastases to the peritoneum occur in $55-60 \%$ of patients with gastric cancer and are associated with a 5 -year overall survival of $2 \%$. Treatment options for these patients are limited, and targeted therapy or immunotherapy is not available. Rational therapeutic targets have yet to be found.In this review, we present the published literature and our own recent experience in molecular biology to identify important molecules and signalling pathways as well as cellular immunity involved in GC peritoneal metastases.

\section{KEYWORDS}

Gastric cancer, chromosomal abnormalities, immunology, molecular biology, peritoneal carcinomatosis, peritoneal metastases, visceral metastasis 


\section{INTRODUCTION}

Gastric cancer is the fifth most common cancer worldwide, with more than one million new cases of gastric cancer diagnosed each year. According to 2018 GLOBOCAN data, gastric cancer is the third leading cause of cancer death worldwide after lung cancer and colorectal cancer in overall mortality. Gastric cancer is rarely diagnosed until it is at stage 34. The mortality rate from gastric cancer is higher in men. An estimated $55-60 \%$ of patients with gastric cancer develop peritoneal-visceral metastases. Despite the use of modern treatments for gastric cancer, including gastrectomy combined with radiotherapy, chemotherapy or targeted chemo-immune therapy, the disease often progresses, recurs or metastasises, and the overall 5-year survival rate is less than $35 \%$ and only $2 \%$ survive to this date for peritoneal and visceral metastases. The peritoneum is usually an ideal medium for the spread of distant metastases caused by gastric cancer. Malignant ascites can result from carcinoma of the stomach, colon, pancreas and ovaries. Malignant ascites has an extremely poor prognosis and does not require treatment. New technologies in genomics and proteomics, including single-stranded RNA sequencing and cytometry, are becoming increasingly effective and important methods for dealing with metastases. Several review articles on gastric cancer have discussed the genomics of peritoneal malignancies, molecular carcinogenesis and molecular mechanisms of peritoneal dissemination, and the clinical significance of micrometastases found at different anatomical sites in patients with gastric cancer. In this article, we try to present you with the latest new findings in the molecular biology and immunology of gastrointestinal peritoneal metastases in gastric cancer We also suggest new potential strategies to improve outcomes in GC patients with peritoneal metastases. The prognosis of advanced gastric cancer remains extreme. Metastasis is the most fatal feature of malignancy, accounting for over 90\% of tumour-related deaths. Distant metastasis of an organ or tissue is a sign of a poor prognosis in patients with gastric cancer. The origin of the tumour cell, genetic variability, circulatory regime and physiological structure of the metastatic organ determine the specific sites of distant metastases. Gene expression of primary gastric cancer and its metastasis has differences that may contribute to early diagnosis and individualised treatment of metastases. However, the heterogeneity of tumour cells is still unclear in different metastases in gastric cancer, which will be a major focus of future research. In this review, we discuss the basic principles of cancer metastasis, the unique physiological characteristics of different metastatic organs and the expression of different gene/protein functions in primary and metastatic gastric cancer. Tumour cell heterogeneity is still unclear in various gastric cancer metastases, which will be a major focus of future research.

The mutation - genetic mutations with specific changes in the gene have been found in patients with gastric cancer. In rare cases ( $\leq 2 \%)$, gastric cancer has a familial origin. Hereditary predisposition to gastric cancer was retested. $37 \%$ had a first-degree relative with gastric cancer, and almost 90\% had a family history of first- and/or second-degree relatives Hereditary diffuse gastric cancer is inherited in an autosomal dominant pattern and is caused by a mutation in the cadherin 1 gene $(\mathrm{CDH} 1$, i.e. E-cadherin). $\mathrm{CDH}_{1}$ function is often lost in various epithelial tumours, and loss of normal intercellular connections promotes cancer invasion and metastasis, and is associated with 
several types of cancer, including gastric cancer. Most of the genetic mutations identified in gastric cancer are associated with changes in biological signals such as phosphatidylinositol 3-kinase. Mutations in genes related to $\mathrm{PI}_{3} \mathrm{~K}$ PIK3A, PTEA, AKT3, AKT2 and $A K T 1$, which are seen more frequently with diffuse gastric cancer, and can affect recurrence patterns. Patients with gastric cancer with PIK3CA amplifications are likely to have recurrent disease and are more often both low- and/or undifferentiated, which is characteristic of peritoneal recurrence.

To differentiate clusters of genes that play a key role in peritoneal metastases, one group of researchers performed a comprehensive whole genome and transcriptome sequencing analysis of consensus primary gastric cancer cells and peritoneal metastases from gastric cancer cells. They found 27 mutated genes, of which 19 genes have been described in the Catalogue of Somatic Mutations in the Cancer Database, and eight genes have recently been described in gastric cancer. The signalling pathways have been analysed for gene mutations occurring in both primary cancer and peritoneal metastases. Significant suppression of genes related to morphogenesis, secretion and epithelial muscle development has also been found in both primary cancer and peritoneal metastases compared to chronic gastritis. Recently, a research group characterised the exome, transcriptome and immune landscape in peritoneal carcinomatosis (PC) cells from 43 patients with gastric cancer (13). The complexity of intratumoral PC heterogeneity was revealed by analysing clonal and subclonal genomic architecture. In addition, new molecular "mesenchymal" and "epithelial-like" subtypes were identified by integrating genomic, transcriptomic and immune characteristics into clusters. The 'mesenchymal-like' subtype was associated with resistance to therapy after peritoneal carcinomatosis Epithelial-mesenchymal metaplasia (EMM) - in which cells lose their epithelial characteristics and acquire mesenchymal characteristics, is associated with various tumour functions, malignant progression, tumour stemness, tumour cell migration, intravascular invasion, metastasis and resistance to therapy asparaginyl endopeptidase (AEP), a lysosomal protease, potentially plays a role in epithelialmesenchymal metaplasia. Asparaginyl endopeptidase is overexpressed in gastric cancer and is expressed at higher levels in PC than in primary gastric cancer. Interestingly, the proliferative, invasive and metastatic ability of gastric cancer cells is inhibited and the subG1 cell population is increased. Asparaginyl endopeptidase is also associated with a significant decrease in transcription factor expression, increased expression of the epithelial marker E-cadherin and, in contrast, decreased expression of mesenchymal markers, $\mathrm{N}$-cadherin, $\beta$-catenin and vimentin. These results indicated that asparaginyl endopeptidase may contribute to invasion and metastasis by modulating EMM. A further study revealed a significant reduction in phosphorylation of AKT and MAPK signalling pathways in gastric cancer cells.

\section{Other proteins involved in gastric cancer with PC}

Different proteins influence the aggressiveness of GC progression and PC metastasis. Protein markers of peritoneal dissemination include secondaries to the gastric wall (EpCAM, E-cadherin), survival and proliferation (HIF-1a), adhesion and 
secondaries to the mesothelium (ITGA, MELK), and expansion and angiogenesis (VEGF). These factors include the PI3K/Art, MAPK/ERK and angiogenesis signalling pathways. Additional molecules involved in gastric carcinogenesis and peritoneal metastases are described below.

Mucins - Members of the mucin family are glycosylated membrane-bound proteins produced in epithelial cells that act as cell signalling and chemical barrier formation. The role of some mucins in gastric cancer and metastasis has recently been well summarised in a review. Mucins often play an inhibitory role. Overexpression of selective mucins is correlated between clinicopathological profiles and patient survival during gastric carcinogenesis. MUC4 is involved in tumour growth and metastasis. IL-11 promotes the transcription of MUC 1 in a co-culture of cancerassociated fibroblasts with gastric cancer cells by activating STAT3, thereby increasing the ability of gastric cancer to metastasise. Different mucins in combination with other markers are used to identify gastric cancer phenotypes.

\section{Factors caused by hypoxia}

Hypoxia-inducible factor (HIF-1) regulates many physiological pathways, such as the regulation of haemopoietic stem cells, cell proliferation, survival, apoptosis, angiogenesis, glucose metabolism and immune cell. Recently, HIF-1a-regulated genes have been found to regulate procollagenlysine-2-oxoglutarate-5-dioxygenase, and expression levels of both HIF-1a and PLOD2 have been implicated in hypoxia. In turn, these elevated PLOD2 levels were associated with peritoneal dissemination and poor survival in patients with gastric cancer. HIF-2a promotes migration, invasion and metastasis in both in vitro and in vivo gastric cancer cells, and overexpression of HIF-2a enhances surfavin, cyclin D1. The cell cycle regulators cyclin D1 and cyclin E2 are highly expressed in gastric carcinoma and are associated with clinical parameters. Cyclin D, as a diagnostic biomarker, has shown significantly higher sensitivity and specificity than cyclin E2. Nevertheless, the differential expression of these cyclins is an early event of gastric carcinogenesis and could be a diagnostic biomarker for early detection of gastric cancer miRNAs have been widely studied in recent years in cancer research and diagnosis. Oncogenic miRNAs activate oncogenic pathways or inhibit tumour suppressor genes, while suppressive miRNAs eliminate the function of oncogenic genes. A single miRNA can target multiple genes or pathways through post-translational mechanisms. Low levels of tumour-suppressive miRNA are associated with signs of cancer aggressiveness, such as cancer proliferation or tumour metastasis, while high levels of tumour-suppressive miRNA reduce cancer growth in vivo and in vitro. Whole-genome transcriptional profiling of miRNA-based signatures was examined in primary gastric cancer tissue from patients with PC. Three miRNAs (miR-30a-5p, -659-3P and -3917) were significantly overexpressed in PC patients, which correlated closely with poor prognosis. The combination of this miRNA signature and the Borman macroscopic type suggests a potentially accurate approach for PC detection.

\section{Suppression of miRNA}

Studies on suppressive miRNA offer potential treatment strategies for gastric cancer. miR$148 \mathrm{a}$ has been shown to reduce tumour proliferation, metastasis, invasiveness, 
migration and adhesive activity of gastric cancer cells through its regulated network of interactions with proteins. Furthermore, miR26a was found to directly target fibroblast growth factor 9 (FGF9), whose overexpression in miR-26a-expressing cells could restore miR$26 a$ invasion and growth defects. Further experiments confirmed that overexpression of miR-148a and miR-26a significantly inhibited cell proliferation, migration, invasion and colony formation as well as induced apoptosis compared to the negative control group. The miR-29 family acts as tumour suppressors, affecting the CCND2 and matrix metalloproteinase-2 genes in gastric cancer (45). miR29a-3p suppressed hyaluronan synthase 3 (HAS3) expression and suppressed gastric cancer proliferation and metastasis. Suppression of miR-198 was characterised in gastric cancer tissues. Restoration of miR-198 expression attenuates gastric cancer cell proliferation and colony formation, while causing significant arrest of $\mathrm{G} 0 / \mathrm{G} 1$. In addition, combination therapy with cisplatin and miR-198 caused stronger antitumour effects than treatment with cisplatin monotherapy. The miR-198/FGFR1 axis plays an important role in gastric cancer proliferation and apoptosis, microRNA-140 expression was significantly reduced in $\mathrm{H}$. pylori positive gastric cancer.

Exosomes obtained from malignant ascites from patients with gastric cancer and gastric cancer cells have been investigated as potential biomarkers of gastric cancer. Exosomal miR-181b-5p in combination with carcinoembryonic antigen (CEA) levels was found to distinguish between benign and gastric ascites cancer. Exosome miRNA are highly expressed in plasma and PC and play a key role in cancer progression. Twenty-nine differentially expressed exosome miRNAs have been found to enhance EMT signalling and initiate peritoneal metastases in vitro and in vivo In addition, 12 miRNAs, including miR-760, miR-6821-5p, etc. D., have been predicted for their target genes, which are involved in TGF- $\beta$, Wnt, Notch and PI3K-AKT signalling pathways and play a role in patients with gastric cancer with peritoneal metastases. These miRNAtranscript interactions e. Provide a new perspective on the mechanisms of peritoneal spread in gastric cancer. Thus, exosomal miRNA represent potential biomarkers or targets for patients with gastric cancer with peritoneal metastases.

\section{Innate and adaptive immune cells in peritoneal metastases from gastric cancer}

Macrophages $(\mathbf{M} \varphi)$ - neutrophils, dendritic cells (DC) and natural killer cells (NK) are involved in innate immunity through direct protection against second gastrointestinal pathogens and cancer cells and initiate adaptive immune systems.T- and Blymphocytes act as key 'soldiers' in adaptive responses through interaction with $D C$, monocytes and $M \varphi$. In general, the basic functional and phenotypic characteristics of these mononuclear phagocytes differ and often overlap.

NK cells - often devoid of antigen-specific cell surface receptors, but soderaque gastric granules, perforin and proteinases such as granzyme $\mathrm{B}$, which induce granule-mediated cytolytic apoptosis or osmotic lysis of target cells. NK cells express activating receptors such as NKG2D and the natural cytotoxicity receptor family (NKp30, NKp46 and NKp44) and recognize many tumor types. NK cells also interact with DC, $M \varphi$ and T cells and contribute to anti-tumour adaptive immune responses by 
secreting cytokines such as IL-2, IL-12, IL-15, IL18 and CCR5. NK cells contribute to the immune surveillance of primary tumour formation, tumour recurrence and metastasis dysfunction in mouse models and patients with gastric cancer. However, NK cells show a suppressive phenotype with fewer activating receptors and higher PD-1 expression in patients with gastric cancer. In addition, NK cells secrete fewer types of cytokines and showed reduced ability to secrete perforins and granzymes. Meanwhile, gastric cancer cells reduce expression of NKG2D, MICA/B and ULBP ligands as well as the $\mathrm{NKp} 30$ ligand, $\mathrm{B} 7 \mathrm{H} 6$, to avoid NK cell-mediated innate immunity. Cancer -cells can also secrete certain cytokines, such as IL-10, TGF- $\beta$ and PGE2, to attract myeloid suppressor cells and regulatory $T$ (Treg)-cells to suppress NK cell activity. Two reports describe an approach to enhance the immunogenicity or sensitivity of peritoneal gastric cancer metastases to NK cells. One method is the use of gene therapy using the intercellular adhesion molecule adenoviral vector (ICAM)-2, which has been administered to mice with peritoneal dissemination of skirrose gastric carcinoma. The mice survived significantly longer and many NK cells filtered out peritoneal metastatic lesions, suggesting that ICAM-2 transfection could be an effective gene therapy strategy for peritoneal metastases from gastric cancer. Another method targets malignant IgG either by transfecting gastric cancer cells with Ig $G$ heavy chain miRNA to enhance antibody-dependent cellular cytotoxicity induced by the EGFR antibody and hence inhibit the growth of gastric cancer cells.

\section{Chimeric antigen receptor T cells}

Chimeric antigen receptor (CAR)-T-cell-based cancer immunotherapy is a promising new alternative for the treatment of recurrent lymphoblastic leukaemia, multiple myeloma and lymphoma by inducing direct cancer killing. Specific CAR-T cells are genetically engineered from patients' $T$ cells and can secrete cytokines, produce specific molecules and exhibit strong cytotoxicity against a wide range of cancer cells. The application of CAR-T cell technology in the treatment of solid tumours is a "hot spot" of current research. Cancer cells expressing NKG2DL are very sensitive to destruction by NK cells. Targeting NKG2DL with engineered $T$ cells expressing chimeric NKG2D (chNKG2D) has been shown to induce tumour elimination and long-term tumour-free survival in mouse models. In addition, the extracellular domain of NKG2D was engineered in human peripheral blood $T$ cells, containing the gastric intracellular domain $\mathrm{CD}_{28}$ in tandem with CD3zeta to enhance $T$ cell activation. This chNKG2D CAR expressed on T cells allows T cells to specifically recognize NKG2DL on the surface of human gastric cancer cell lines and primary cancer cells originating from ascites expressing surface NKG2DL. Thus, chNKG2D receptor-modified $T$ cells represent a potential therapeutic strategy for NKG2DL-expressing gastric cancer with peritoneal metastases.

A new type of CAR-T cell is needed to break through the bottleneck of treatment for solid tumours. A new potential target in gastric cancer is the human trophoblast cell surface antigen 2 (Trop2), which is frequently expressed in various epithelial tissue tumours including gastric cancer cells and contributes to malignant biological behaviour including lymph node and distant metastases. Similarly, PD-L1 is expressed at significantly higher levels in gastric cancer tissues and lymphoid tissues 
of gastric cancer metastases is associated with poor survival prognosis .

\section{Immune checkpoint molecules in peritoneal metastases of gastric cancer}

Immunological checkpoint inhibitors affect various neglected forms of cancer. However, their basic research and clinical trials have not yet established peritoneal metastasis from gastric cancer. The immune microenvironment of the tumour is altered after neoadjuvant chemotherapy for locally advanced gastric cancer. A panel of immune cells infiltrating the tumour and lymphocyte checkpoint molecules was examined in 60 patients with advanced gastric cancer. Overall expression levels of CD4, CD8, PD1, PD-L1 and TIM-3 were significantly increased with post-neoadjuvant chemotherapy compared to pre-treatment levels. Individually, in contrast to increased marker expression in most patients, a minority of patients had decreased expression of these markers plus FOXP3. Changes in PD-1, PD-L1 and TIM-3 expression between pre- and postneoadjuvant chemotherapy showed strong positive pairwise correlations with each other. Multivariate analysis demonstrated that high levels of CD8, PD-1 and PD-L1 after neoadjuvant chemotherapy were favourable prognostic factors for overall survival. The overall increased expression of checkpoint molecules and infiltration of immune cells may indicate a dynamic bidirectional shift in the tumour microenvironment during neoadjuvant chemotherapy for gastric cancer. These data provide the possibility of using chemotherapy in combination with immunotherapy or even dual checkpoint blockade in locally advanced gastric cancer.

\section{Cytokine profiles in peritoneal metastases}

IL-2 - Immune cells secrete cytokines in response to pathogenic inflammation, infection and cancer. IL-2 is a secreted mediator that is important for the proliferation of $\mathrm{T}$ - and B-lymphocytes and for the activation of cytotoxic T-lymphocytes, helper Tlymphocytes, NK cells and lymphokine activated killer cells. The administration of IL-2 in some tumour models has been shown to contribute to anti-tumour immunity where it is suggested that IL-2 facilitates anergic blockade of T cells , CD80, as a CD28 ligand, plays a key role in the induction of cell-mediated immune responses and the co-expression of CD80 and IL-2 in T cells reduces carcinogenicity in some types of malignancies .

Interferon beta (IFN- $\beta$ ) - has been used to treat several types of cancer in preclinical and clinical studies. The essential nature of interferonactivated immune signal transduction and its role in stimulating immune effector cell functions to prevent or eradicate tumours has been established in mouse models. New engineered forms of human and murine IFN- $\beta$, named PEG-hIFN- $\beta$ and PEG-mIFN- $\beta$, in which IFN- $\beta$ is conjugated to polyethylene glycol, were developed. These IFN- $\beta$ molecules retained antiviral activity similar to natural IFN$\beta$ in vitro and exhibited improved in vivo pharmacokinetics.. Interestingly, PEG-hIFN- $\beta$ directly suppressed VEGF165-induced hyperpermeability in human vascular endothelial cells. PEG-mIFN- $\beta$ significantly suppressed ascitic fluid accumulation and peritoneal membrane vascular permeability in xenograft models of ovarian cancer and gastric cancer in mice. PEG-mIFN- $\beta$ enhanced the expression of a number of cell adhesionrelated molecules in vitro. These data show that PEG-ylated long-acting hIFN- $\beta$ maintains vascular integrity and is a therapeutic agent for 
the effective treatment of malignant ascites. The cytokine TGF- $\beta$ has long been studied in inflammation and oncogenesis. Altered TGF- $\beta$ signalling pathway is associated with liver and gastrointestinal cancers. The function of TGF- $\beta$ is context-dependent; it aids gastric subdermal hemostasis by regulating cell growth, differentiation and survival; it controls tumours at early stages by promoting cell cycle arrest and apoptosis; and it allows tumour invasion and metastasis at later stages. Although TGF- $\beta$ acts as a tumour suppressor in the gastrointestinal tract, it can also act as a tumour promoter. These dual effects of TGF- $\beta$ are a major obstacle to the development of drugs targeting this pathway, and several drugs targeting the TGF- $\beta$ pathway have proven effective in clinical trials. Thus, the identification of new strategies targeting specific components of this pathway will lead to improved outcomes for lethal liver and gastrointestinal cancers. Significantly increased expression of TGF- $\beta 1$ was found when both gastric tumour cells and mesothelial cells were co-cultured compared to individual culturing conditions. Serum-free conditioned medium from human gastric cancer cells significantly induced extracellular matrix expression in vitro and in vivo, and oncogenicity was higher in mice with peritoneal fibrosis than in mice with normal peritoneum. Blocking of TGF- $\beta 1$ by the peptide P17 partially facilitates the development of peritoneal metastases. These results indicated that TGF- $\beta 1$ plays an important role in the induction of peritoneal fibrosis when gastric cancer interacts with peritoneal fibrosis, which in turn affects the spread of gastric cancer.

\section{CONCLUSIONS}

In summary, existing research has confirmed a broader and deeper understanding of the mechanisms of peritoneal gastric cancer metastasis in molecular biology and immunology. However, we are still in the middle of a full understanding of gastric cancer progression and metastasis. Detailed identification of more molecules and their interactions involved in different signalling pathways of gastric cancer with PC will lead to new strategies for targeting key components of peritoneal gastric cancer metastases, as well as improving specific therapeutics in the clinic.

\section{REFERENCES}

1. Akhmedov R.M., Mirkhojaev I.A., Khamdamov B.Z. Morphostructural changes in the liver in the elderly and old age // Conference proceedings. Journal of Problems of Biology and Medicine. - 2016. - №3,1(90). - C. 18.56.

2. Azimov M. I., Shomurodov K.E. A technique for Cleft Palate Repair. Journal of research in health science. Vol. 1, No. 2, 2018, pp. 56-59

3. Bollen, L., Jacobs, W.C.H., Van der Linden, Y.M. et al. A systematic review of prognostic factors predicting survival in patients with spinal bone metastases. Eur Spine J 27, 799-805 (2018). https://doi.org/10.1007/s00586017-5320-3

4. Crawford, E.D., et al., Challenges and recommendations for early identification of metastatic disease in prostate cancer. Urology, 2014.83 (3): p. 664-9.

5. Lipton, A. The Science and Practice of Bone Health in Oncology: Managing Bone Loss and Metastasis in Patients With Solid Tumors / A. Lipton // J. Nat. Compr. Canc. Netw. - 2009. - Vol. 7. - P. 1-29 
6. $\quad F$ Ruatta, L Derosa, B Escudier, E Colomba, A Guida, G Baciarello, Y Loriot, K Fizazi, L Albiges, European Journal of Cancer. Prognosis of Renal Cell Carcinoma With Bone Metastases: Experience From a Large Cancer Center Eur. J. Cancer 2018 Dec 11; 107 (xx) 7985

7. G. M. HODJIMATOV, K. K. MIRZAEV, D. T. AZIZOV. PHARMACOKINETICS OF ANTIBIOTICS IN EXPERIMENTAL GUNSHOT WOUNDS. A Multidisciplinary Peer Reviewed Journal Research for Revolution ISSN No-2581 - 4230,Is Published Online in Volume-7, Issue2, Feb. - 2021,pp120-123

8. G. M. HODJIMATOV, Kh. Kh. Hamdamov. DIAGNOSTICS AND TREATMENT OF CHOLEDOCHOLITIASIS IN ELDERLY AND SENILE AGE PATIENTS // European Journal of Research Development and Sustainability (EJRDS) Available Online at: https://www.scholarzest.com Vol. 2 No. 3, March 2021, ISSN: 2660-5570

9. Kamalova M. I., Islamov Sh. E., Khaydarov N.K.// MORPHOLOGICAL CHANGES IN BRAIN VESSELS IN ISCHEMIC STROKE. Journal of Biomedicine and Practice 2020, vol. 6, issue 5, pp.280-284

10. Khamdamov B.Z. Indicators of immunocitocine status in purulentnecrotic lesions of the lover extremities in patients with diabetes mellitus.//American Journal of Medicine and Medical Sciences, 202010 (7) 473-478 DOI: 10.5923/j.ajmm.2020.1007.08

11. Musaev U.Y.,Rizaev J.A., Shomurodov K.E. New views on the problem of dysemryogenesis stigmas of dento- mandibular and facial system from the position of their formation in the disability of the population // Central Asian Scientific and Practical Journal "Stomatologiya "2017.-\#3-(68).-P.9-12.

12. Tillyashaikhov MN, Yusupov Sh. Kh., Boyko EV, Valieva RM Criteria for choosing hormonal therapy for patients with advanced prostate cancer // Bulletin of the Tashkent Medical Academy. - 2016. - No. 2 (05). S. 95-97

13. Tillyashaikhov $M N$, Rakhimov NM, Tillyashaikhova RM Evolution of views on the diagnosis and treatment of renal cell cancer // Medical Journal of Uzbekistan. - Tashkent, 2018.- №4. - S. 51-55.

14. Tillyashayakhov M. N., Rahimov N. M., Tillyashayakhova R. M. Long-term results of modified surgical access to regional lymph nodes and main vessels in the treatment of renal cell carcinoma // The USA Journals Volume 03 Issue 022021174 The American Journal of Medical Sciences and Pharmaceutical Research (ISSN - 2689-1026) Published: February 28, 2021 | Pages: 161-174 Doi: https://doi.org/10.37547/TAJMSPR/Vol ume03Issue02-24

15. : Rahimov N, Shakhanova Sh. Development of new approaches in treatment of metastatic renal cell carcinoma // Journal of research in health science Volume 5-6 issue. 4 2020, pp. 82-95 ISSN 2523-1251 (Online), p 82-95.

16. Saidkulov B, Abduraxmonov A, Rahimov N. Recurrent ovarian cancer: mechanisms of development of peritoneal malignant ascites $/ /$. European Journal of Molecular \& 
Clinical Medicine ISSN 2515-8260

Volume 7, Issue 2, 2020, p 2423-2428

17. Shakhanova Sh.Sh., Djuraev M.D., Rakhimov N.M., Karimova M.N., Asatulayev A.F., (2021). Determination Of Risk Parameters In The Detection of Asymptomatic Bone Metastases of Kidney And Prostate Cancer. The American Journal of Medical Sciences and Pharmaceutical Research, 3(02), 161-174.

18. Ziyadullaev Sh, Elmamatov O, F Raufov Cytogenetic and immunological alterations of recurrent bladder cancer // European Journal of Molecular \& Clinical Medicine 2020, Volume 7, Issue 2, p 1877-1883. 